\title{
On the Optimal Dividend Problem for Insurance Risk Models with Surplus-Dependent Premiums
}

\author{
Ewa Marciniak $^{1} \cdot$ Zbigniew Palmowski $^{2}$
}

Received: 18 December 2014 / Accepted: 11 May 2015 / Published online: 27 May 2015

C The Author(s) 2015. This article is published with open access at Springerlink.com

\begin{abstract}
This paper concerns an optimal dividend distribution problem for an insurance company with surplus-dependent premium. In the absence of dividend payments, such a risk process is a particular case of so-called piecewise deterministic Markov processes. The control mechanism chooses the size of dividend payments. The objective consists in maximizing the sum of the expected cumulative discounted dividend payments received until the time of ruin and a penalty payment at the time of ruin, which is an increasing function of the size of the shortfall at ruin. A complete solution is presented to the corresponding stochastic control problem. We identify the associated Hamilton-Jacobi-Bellman equation and find necessary and sufficient conditions for optimality of a single dividend-band strategy, in terms of particular Gerber-Shiu functions. A number of concrete examples are analyzed.
\end{abstract}

Keywords Optimal strategy · PDMP · Barrier strategy · Integro-differential HJB equation $\cdot$ Gerber-Shiu function $\cdot$ Stochastic controls

Mathematics Subject Classification $\quad 60 \mathrm{G} 51 \cdot 60 \mathrm{G} 50 \cdot 60 \mathrm{~K} 25 \cdot 93 \mathrm{E} 20$

\section{Introduction}

In classical collective risk theory, the surplus of an insurance company is described by the Cramér-Lundberg model. Under the assumption that the premium income per unit time is larger than the average amount claimed, the surplus in the Cramér-Lundberg

\footnotetext{
Zbigniew Palmowski

zbigniew.palmowski@gmail.com

1 AGH University of Science and Technology, Kraków, Poland

2 University of Wrocław, Wrocław, Poland
} 
model has positive first moment and has therefore the unrealistic property that it converges to infinity with probability one. In answer to this objection, De Finetti [1] introduced the dividend barrier model, in which all surpluses above a given level are transferred to a beneficiary, and raised the question of optimizing this barrier. In the mathematical finance and actuarial literature, there is a good deal of work being done on dividend barrier models and the problem of finding an optimal policy of paying dividends. Gerber and Shiu [2] and Jeanblanc and Shiryaev [3] consider the optimal dividend problem in a Brownian setting. Irbäck [4] and Zhou [5] study constant barriers. Asmussen et al. [6] investigate excess-of-loss reinsurance and dividend distribution policies in a diffusion setting. Azcue and Muler [7] take a viscosity approach to investigate optimal reinsurance and dividend policies in the Cramér-Lundberg model using a Hamilton-Jacobi-Bellman (HJB) system of equations. Avram et al. [8,9], Kyprianou and Palmowski [11], Loeffen [12,13], Loeffen and Renaud [14] and many other authors analyze the Lévy risk processes set up from the probabilistic point of view.

In this paper, we shall approach the dividend problem for a reserve-dependent risk process using the theory of piecewise deterministic Markov processes (PDMP). We also take into account the "severity" of ruin and therefore we consider the socalled Gerber-Shiu penalty function (see, e.g., Schmidli [15] or Avram et al. [9] and references therein). For this setup, without transaction costs, we find the corresponding HJB system. We analyze the barrier strategy for which all surpluses above a given level are transferred to dividends. In particular, we find necessary and sufficient conditions for the barrier strategy to be optimal.

We believe that PDMP models can better describe the situation of an insurance company, since, for example, they can invest the surplus into a bond with a fixed interest rate. Such a situation is described by a PDMP model with a linear premium (see [10]).

The paper is organized as follows. In Sect. 2, we introduce the basic notation and we describe the model we deal with. Section 3 is dedicated to the related one-sided and two-sided problems. In Sect. 4, we present the Verification Theorem, necessary and sufficient conditions for the barrier strategy to be optimal. In Sect. 6, we give all the proofs. Section 5 and 7 are devoted to some examples and concluding remarks.

\section{The Model}

In this paper, we assume that the surplus $R$ of an insurance company (without payment of dividends) with an initial capital $x$ is described by the following differential equation:

$$
R_{t}=x+\int_{0}^{t} p\left(R_{s}\right) \mathrm{d} s-\sum_{k=1}^{N(t)} C_{k},
$$

where $p$ is a given deterministic positive premium function, $\left\{C_{k}\right\}_{i=1}^{\infty}$ is a sequence of i.i.d. positive random variables with d.f. $F$ representing the claims and $N$ is an 
independent Poisson process with intensity $\lambda$ modeling the times at which the claims occur.

We assume that $R_{t} \rightarrow \infty$ a.s., $\mathbb{E} C<\infty$ for a generic claim $C$, and the premium rate $p$ is monotone, absolutely continuous and satisfies the following "speed condition":

$$
\int_{0}^{\infty} \mathrm{e}^{-q t} p\left(r_{t}^{x}\right) \mathrm{d} t \leq A x+B
$$

for some constants $A, B \geq 0$ and a function $r^{x}$ satisfying the equation

$$
r_{t}^{x}=x+\int_{0}^{t} p\left(r_{s}^{x}\right) \mathrm{d} s .
$$

Note that $r^{x}$ describes a deterministic trajectory of $R$ along which no claims appear.

Remark 2.1 Constant and linear premium functions satisfy the above assumptions. For a constant premium function, we obtain the classical Cramér-Lundberg model.

To approach the dividend problem, we consider the regulated risk process satisfying the following stochastic differential equation:

$$
X_{t}^{\pi}=x+\int_{0}^{t} p\left(X_{s}^{\pi}\right) \mathrm{d} s-\sum_{k=1}^{N(t)} C_{k}-L_{t}^{\pi},
$$

where $\pi$ denotes a strategy chosen from the class $\Pi$ of all "admissible" dividend controls resulting in the cumulative amounts of dividends $L_{t}^{\pi}$ paid up to time $t$. Note that ruin may be either exogeneous or endogeneous (i.e., caused by a claim or by a dividend payment). A dividend strategy is admissible, if ruin is always exogeneous or, more precisely, an admissible dividend strategy $L^{\pi}=\left\{L_{t}^{\pi}, t \in \mathbb{R}_{+}\right\}$is a rightcontinuous stochastic process, adapted to the natural filtration of the risk process $R$ that satisfies the usual conditions, and such that, at any time preceding the epoch of ruin, the dividend payment is smaller than the size of the available reserves $\left(L_{t}^{\pi}-L_{t-}^{\pi}<X_{t-}^{\pi}\right)$.

The object of interest is the discounted cumulative dividend paid up to the ruin time:

$$
D(\pi):=\int_{0}^{T^{\pi}} \mathrm{e}^{-q t} \mathrm{~d} L_{t}^{\pi},
$$

where $T^{\pi}:=\inf \left\{t \geq 0: X_{t}^{\pi}<0\right\}$ is the ruin time and $q \geq 0$ is a given discount rate. Note that unless it is necessary we will write $T$ instead of $T^{\pi}$ to simplify the notation. The objective is to maximize $\mathbb{E}_{x} D(\pi)$, where $\mathbb{E}_{x}$ is the expectation with respect to $\mathbb{P}_{x}(\cdot)=\mathbb{P}\left(\cdot \mid X_{0}^{\pi}=x\right)$. We will use the notation $\mathbb{P}_{0}=\mathbb{P}$ and $\mathbb{E}_{0}=\mathbb{E}$.

To take into account the "severity" of ruin, we also consider the so-called GerberShiu penalty function

$$
\mathbb{E}_{x}\left[\mathrm{e}^{-q T} w\left(X_{T}^{\pi}\right) \mathbb{I}_{\{T<\infty\}}\right]
$$


for some general non-positive penalty function $w$ satisfying the integrability condition

$$
\sup _{y \geq 0} \mathbb{E}\left[-w\left(y-C_{1}\right) \mid C_{1}>y\right]<\infty
$$

Note that for $q=0$ and $w=-1$, we derive the ruin probability.

The dividend problem consists in finding the so-called value function $v$ given by

$$
v(x):=\sup _{\pi \in \Pi} v_{\pi}(x)
$$

where

$$
v_{\pi}(x):=\mathbb{E}_{x}\left[\int_{0}^{T} \mathrm{e}^{-q t} \mathrm{~d} L_{t}^{\pi}+e^{-q T} w\left(X_{T}^{\pi}\right) \mathbb{I}_{\{T<\infty\}}\right]
$$

and the optimal strategy $\pi_{*} \in \Pi$ such that

$$
v(x)=v_{\pi_{*}}(x) \text { for all } x \geq 0
$$

\section{Preliminaries}

For the solution of the dividend problem, two functions, $W_{q}$ and $G_{q, w}$, are crucial. They are related to two-sided and one-sided exit problems for $R$ :

$$
\begin{aligned}
& \mathbb{E}_{x}\left[\mathrm{e}^{-q \tau_{a}^{+}} \mathbb{I}_{\left\{\tau_{a}^{+}<\tau_{0}^{-}\right\}}\right]=\frac{W_{q}(x)}{W_{q}(a)}, \\
& G_{q, w}(x):=\mathbb{E}_{x}\left[\mathrm{e}^{-q \tau_{0}^{-}} w\left(R_{\tau_{0}^{-}}\right) \mathbb{I}_{\left\{\tau_{0}^{-}<\infty\right\}}\right],
\end{aligned}
$$

where $x \in[0, a], \tau_{a}^{+}:=\inf \left\{t \geq 0: R_{t} \geq a\right\}$ and $\tau_{0}^{-}:=\inf \left\{t \geq 0: R_{t}<0\right\}$. From now on, we will assume the existence of the function $W_{q}$, which follows for example from the existence of the following limit: $W_{q}(x)=\lim _{y \rightarrow \infty} \mathbb{E}_{x}\left[\mathrm{e}^{-q \tau_{y}^{+}} \mathbb{I}_{\left\{\tau_{y}^{+}<\tau_{0}^{-}\right\}}\right] / \mathbb{E}\left[\mathrm{e}^{-q \tau_{y}^{+}}\right.$ $\left.\mathbb{I}_{\left\{\tau_{y}^{+}<\tau_{0}^{-}\right\}}\right]$. Indeed, using the strong Markov property of $R$ that has only negative jumps, we derive

$$
\begin{aligned}
W_{q}(x) & =\lim _{y \rightarrow \infty} \frac{\mathbb{E}_{x}\left[\mathrm{e}^{-q \tau_{a}^{+}} \mathbb{I}_{\left\{\tau_{a}^{+}<\tau_{0}^{-}\right\}}\right] \mathbb{E}_{a}\left[\mathrm{e}^{-q \tau_{y}^{+}} \mathbb{I}_{\left\{\tau_{y}^{+}<\tau_{0}^{-}\right\}}\right]}{\mathbb{E}\left[\mathrm{e}^{-q \tau_{y}^{+}} \mathbb{I}_{\left\{\tau_{y}^{+}<\tau_{0}^{-}\right\}}\right]} \\
& =\mathbb{E}_{x}\left[\mathrm{e}^{-q \tau_{a}^{+}} \mathbb{I}_{\left\{\tau_{a}^{+}<\tau_{0}^{-}\right\}}\right] W_{q}(a),
\end{aligned}
$$

which gives the required identity (7).

For the properties of the function $G_{q, w}$, we refer the reader to [18], where numerous examples are studied. 


\section{Main Results}

To prove the optimality of a particular strategy $\pi$ among all admissible strategies $\Pi$ for the dividend problem (5), we consider the following Hamilton-Jacobi-Bellman (HJB) system:

$$
\begin{aligned}
& \max \left\{\mathcal{A} m(x)-q m(x), 1-m^{\prime}(x)\right\} \leq 0 \text { for all } x>0, \\
& m(x)=w(x) \text { for all } x<0,
\end{aligned}
$$

where $\mathcal{A}$ is the full generator of $R$,

$$
\mathcal{A} m(x)=p(x) m^{\prime}(x)+\lambda \int_{0}^{\infty}(m(x-y)-m(x)) \mathrm{d} F(y),
$$

acting on absolutely continuous functions $m$ such that

$$
\mathbb{E}\left[\sum_{\sigma_{i} \leq t}\left|m\left(R_{\sigma_{i}}\right)-m\left(R_{\sigma_{i}-}\right)\right|\right]<\infty \quad \text { for any } t \geq 0
$$

where $\left\{\sigma_{i}\right\}_{i \in \mathbb{N} \cup\{0\}}$ denotes the times at which the claims occur (see Davis [16] and Rolski et al. [17]). In this case, $m^{\prime}$ denotes the density of $m$. Note that any function, which is absolutely continuous and ultimately dominated by an affine function, is in the domain of the full generator $\mathcal{A}$, as a consequence of the assumption that $\mathbb{E} C_{1}<\infty$. Recall that, for any function $m$ from the domain of $\mathcal{A}$, the process

$$
\left\{\mathrm{e}^{-q t} m\left(R_{t}\right)-\int_{0}^{t} \mathrm{e}^{-q s}(\mathcal{A}-q) m\left(R_{S}\right) \mathrm{d} s, t \geq 0\right\}
$$

is a martingale.

Theorem 4.1 (Verification Theorem) Let $\pi$ be an admissible dividend strategy such that $v_{\pi}$ is absolutely continuous and ultimately dominated by some affine function. If (9) holds for $v_{\pi}$ then $v_{\pi}(x)=v(x)$ for all $x \geq 0$.

The proof of all theorems given here will be given in Sect. 6 .

Lemma 4.1 Assume that the distribution function (d.f.) $F$ of the claim size is absolutely continuous. Then the functions $W_{q}$ and $G_{q, w}$ are continuously differentiable for all $x \geq 0$.

From now on, we assume that the claim size distribution is absolutely continuous with a density $f$.

We will focus on the so-called barrier policy $\pi_{a}$ transferring all surpluses above a given level $a$ to dividends. 
Theorem 4.2 We have

$$
v_{a}(x):=v_{\pi_{a}}(x)= \begin{cases}\frac{W_{q}(x)}{W_{q}^{\prime}(a)}\left(1-G_{q, w}^{\prime}(a)\right)+G_{q, w}(x), & x \leq a, \\ x-a+v_{a}(a), & x>a .\end{cases}
$$

Moreover, $v_{a}$ is continuously differentiable for all $x \geq 0$.

Let

$$
H_{q}^{\prime}(y):=\frac{1-G_{q, w}^{\prime}(y)}{W_{q}^{\prime}(y)} .
$$

Define now a candidate for the optimal dividend barrier by

$$
a^{*}:=\sup \left\{a \geq 0: H_{q}^{\prime}(a) \geq H_{q}^{\prime}(x) \text { for all } x \geq 0\right\},
$$

where $H_{q}^{\prime}(0)=\lim _{x \downarrow 0} H_{q}^{\prime}(x)$.

Finally, using the above two theorems, we can give necessary and sufficient conditions for the barrier strategy to be optimal.

Theorem 4.3 The value function under the barrier strategy $\pi_{a^{*}}$ is in the domain of the full generator $\mathcal{A}$. The barrier policy $\pi_{a^{*}}$ is optimal and $v_{a^{*}}(x)=v(x)$ for all $x \geq 0$ if and only if

$$
(\mathcal{A}-q) v_{a^{*}}(x) \leq 0 \quad \text { for all } x>a^{*}
$$

Theorem 4.4 Suppose that

$$
H_{q}^{\prime}(a) \geq H_{q}^{\prime}(b) \quad \text { for all } a^{*} \leq a \leq b
$$

Then the barrier strategy at $a^{*}$ is optimal, that is, $v(x)=v_{a^{*}}(x)$ for all $x \geq 0$.

Theorem 4.5 Suppose that $f$ is convex and $p$ is concave. Then the barrier strategy at $a^{*}$ is optimal, that is, $v(x)=v_{a^{*}}(x)$ for all $x \geq 0$.

Theorem 4.6 Consider the problem without the penalty function $(w \equiv 0)$. Suppose that $f$ is decreasing and

$$
p^{\prime}(x) \leq q+\lambda, \quad x \geq a^{*},
$$

where $p^{\prime}$ is the density of the premium rate $p$. Then the barrier strategy at $a^{*}$ is optimal, that is, $v(x)=v_{a^{*}}(x)$ for all $x \geq 0$. 


\section{Examples}

In this section, we will assume that the premium function $p$ is differentiable and the generic claim size has a density $f$ with a rational Laplace transform. That is, there exists $m \in \mathbb{N}$ and constants $\left\{\beta_{i}\right\}_{i=0}^{m-1}$ such that the density $f$ satisfies the following LODE:

$$
\mathcal{L}\left(\frac{\mathrm{d}}{\mathrm{d} y}\right) f(y)=0
$$

with initial conditions $f^{(k)}(0)=0(k=0, \ldots, m-2)$, where

$$
\mathcal{L}(x)=x^{m}+\beta_{m-1} x^{m-1}+\cdots+\beta_{0} .
$$

Note that by Theorem 4.5 if we take $p$ concave then the barrier strategy is optimal for an exponential claim size (in this case $\mathcal{L}(x)=x+\mu$ ). From Lemma 4.1 and its proof, it follows that if the claim size distribution is absolutely continuous then $W_{q}$, $G_{q, w}$ and $v_{a^{*}}$ are differentiable and satisfy

$$
\mathcal{A} W_{q}(x)=q W_{q}(x) \text { for } x \geq 0, \quad W_{q}(x)=0 \text { for } x<0,
$$

and

$$
\mathcal{A} G_{q, w}(x)=q G_{q, w}(x) \text { for } x \geq 0, \quad G_{q, w}(x)=w(x) \text { for } x<0 .
$$

Our goal will be to find the value function $v$ for a few examples of premium functions. The Gerber-Shiu function $G_{q, w}$ was determined in Albrecher et al. [18]. One can prove that if $G_{q, w}$ is differentiable then in fact $G_{q, w} \in \mathcal{C}^{m+1}$ (see [17]). The same holds for $W_{q}$. Albrecher et al. [18] proved that $G_{q, w}$ satisfies the following LODE with variable coefficients of order $m+1$ :

$$
\mathbf{T} G_{q, w}(x)=u(x)
$$

with the differential operator

$$
\mathbf{T}:=\mathcal{L}\left(\frac{\mathrm{d}}{\mathrm{d} x}\right)\left(q-p(x) \frac{\mathrm{d}}{\mathrm{d} x}+\lambda\right)-\lambda \beta_{0}
$$

and the right-hand side

$$
u(x):=\lambda \mathcal{L}\left(\frac{\mathrm{d}}{\mathrm{d} x}\right) \omega(x),
$$

where $\omega(x):=\int_{x}^{\infty} w(x-z) \mathrm{d} F(z)$. In general, the main idea of solving the above equation is to find stable solutions $s_{k}$ of the fundamental system for (16) (that is, those vanishing at infinity) and then use the representation

$$
G_{q, w}(x)=\gamma_{1} s_{1}(x)+\cdots+\gamma_{m} s_{m}(x)+G u(x)
$$


where $G$ is the Green operator and the constants $\gamma_{i}$ can be computed from the initial conditions. Moreover, the form of the Green operator is found in [18, Thm.3.4].

If the claim size has exponential distribution with intensity $\mu$ then we can prove that $G_{q, w}$ solves the following ODE:

$$
\left(\frac{\mathrm{d}^{2}}{\mathrm{~d} u^{2}}+\left(\mu+\frac{p^{\prime}(x)}{p(x)}-\frac{\lambda+q}{p(x)}\right) \frac{\mathrm{d}}{\mathrm{d} u}-\frac{q \mu}{p(x)}\right) G_{q, w}(x)=u(x),
$$

with $u(x)=-\frac{\lambda}{p(x)}\left(\frac{\mathrm{d}}{\mathrm{d} u}+\mu\right) \omega(x)$. This allows one to find $G_{q, w}$ explicitly.

Moreover, note that (14) is a Gerber-Shiu function with zero penalty function. In contrast to the one from (7), we now have $\lim _{x \rightarrow \infty} W_{q}(x)=+\infty$. This means that the optimal value function under mild conditions is a linear combination of two GerberShiu functions: An unstable one that vanishes on the negative half line and tends to infinity at infinity (corresponding to dividend payment, $W_{q}$ in our notation), and a stable one, vanishing at infinity (corresponding to the penalty payment, $G_{q, w}$ in our notation). From [18], we know that $W_{q}$ equals the unstable solution of the fundamental system for (16). One can prove that there exists a unique unstable solution (see [18] for details). In the rest of this section, we will assume that the claim size has exponential distribution with intensity $\mu$.

\subsection{Linear Premium}

We take here $p(x)=c+\epsilon x$. By Theorem 4.5, the barrier strategy at $a^{*}$ is optimal. In this case,

$$
s_{1}(x)=U\left(\frac{q}{\epsilon}+1, \frac{\lambda+q}{\epsilon}+1, \mu x+\frac{\mu c}{\epsilon}\right)(\epsilon x+c)^{(\lambda+q) / \epsilon} \exp (-\mu x)
$$

and

$$
\begin{aligned}
G u(x)= & \frac{\Gamma(q / \epsilon+1)}{\Gamma((q+\lambda) /(1+\epsilon))} \frac{1}{\epsilon}\left(\frac{\mu}{\epsilon}\right)^{(\lambda+q) / \epsilon}(\epsilon x+c)^{(\lambda+q) / \epsilon} \exp \left(-\mu x-\frac{\mu c}{\epsilon}\right) \\
& \times\left(-U(x) \int_{0}^{x} M(v) u(v) \mathrm{d} v-M(x) \int_{x}^{\infty} U(v) u(v) \mathrm{d} v\right. \\
& \left.\quad+\frac{M(0)}{U(0)} U(x) \int_{0}^{\infty} U(v) u(v) \mathrm{d} v\right),
\end{aligned}
$$

where $U(u)$ and $M(u)$ are Kummer functions. This gives

$$
G_{q, w}(x)=s_{1}(x)+G u(x)
$$

$$
\begin{aligned}
& \text { for } u(x)=-\frac{\lambda}{p(x)}\left(\frac{\mathrm{d}}{\mathrm{d} u}+\mu\right) \omega(x) \text {. Moreover, } \\
& \qquad \begin{aligned}
W_{q}(x)= & C_{1} M\left(\frac{q}{\epsilon}+1, \frac{\lambda+q}{\epsilon}+1, \mu x+\frac{\mu c}{\epsilon}\right)(\epsilon x+c)^{(\lambda+q) / \epsilon} \exp (-\mu x) \\
& +C_{2} U\left(\frac{q}{\epsilon}+1, \frac{\lambda+q}{\epsilon}+1, \mu x+\frac{\mu c}{\epsilon}\right)(\epsilon x+c)^{(\lambda+q) / \epsilon} \exp (-\mu x),
\end{aligned}
\end{aligned}
$$


Table 1 Linear premium. Dependence of $q$ on $a^{*}$

Table 2 Linear premium. Dependence of $\mu$ on $a^{*}$

Table 3 Linear premium. Dependence of $\lambda$ on $a^{*}$

Table 4 Rational premium. Dependence of $q$ on $a^{*}$

\begin{tabular}{lccccc}
\hline$\mu=0.3, \epsilon=0.02, \lambda=0.1, c=1$ \\
\hline$q$ & 0.025 & 0.03 & 0.04 & 0.05 & 0.06 \\
$a^{*}$ & 17.82 & 13.42 & 8.42 & 5.33 & 3.18 \\
\hline
\end{tabular}

\begin{tabular}{lllllll}
\hline$q=0.05, \epsilon=0.02, \lambda=0.1, c=1$ \\
\hline$\mu$ & 0.25 & 0.3 & 0.4 & 0.5 & 0.6 & 1.1 \\
$a^{*}$ & 3.97 & 5.33 & 5.92 & 5.7 & 5.3 & 3.72 \\
\hline
\end{tabular}

\begin{tabular}{llllll}
\hline$\mu=0.3, q=0.05, \epsilon=0.02, c=1$ \\
\hline$\lambda$ & 0.05 & 0.12 & 0.15 & 0.17 & 0.2 \\
$a^{*}$ & 4.84 & 5.03 & 4.08 & 3.1 & 1.07 \\
\hline
\end{tabular}

\begin{tabular}{lcrcr}
\hline$\mu=0.3, \lambda=0.1, c=1$ & & & \\
\hline$q$ & 0.005 & 0.01 & 0.015 & 0.02 \\
$a^{*}$ & 37.03 & 23.98 & 17.16 & 12.77 \\
\hline
\end{tabular}

with $C_{1}$ and $C_{2}$ determined by the boundary conditions $W_{q}(0)=1$ and $W_{q}^{\prime}(0)=$ $(\lambda+q) / c$.

Hence we can find the optimal barrier $a^{*}$ by solving $H_{q}^{\prime \prime}\left(a^{*}\right)=0$. In the case of absence of the penalty function, that is, when $w(x)=0$, we can perform some numerical analysis of the values of $a^{*}$. In Tables 1, 2 and 3, we present some values of $a^{*}$ for different parameters.

\subsection{Rational Premium}

In this subsection, we consider the rational premium with $p(x)=c+1 /(1+x)$. One can solve Eq. (14) and find the function $W_{q}$. If we take $w \equiv 0$ then, to get optimality of the barrier strategy using Theorem 4.6, we will assume that $\epsilon \leq q+\lambda$. Thus, in the absence of the penalty function, we can find the values of $a^{*}$ for different parameters. In Tables 4, 5 and 6, we give some results in the case of a rational premium.

Table 5 Rational premium. Dependence of $\mu$ on $a^{*}$

\begin{tabular}{llllc}
\hline$q=0.01, \lambda=0.1, c=1$ & & & \\
\hline$\mu$ & 0.15 & 0.2 & 0.25 & 0.3 \\
$a^{*}$ & 0 & 23.98 & 22.39 & 20.05 \\
\hline
\end{tabular}


Table 6 Rational premium. Dependence of $\lambda$ on $a^{*}$

\begin{tabular}{lccccr}
\hline$q=0.01, \mu=0.3, c=1$ \\
\hline$\lambda$ & 0.05 & 0.12 & 0.15 & 0.2 & 0.25 \\
$a^{*}$ & 17.73 & 20.55 & 20.8 & 19.16 & 13.29 \\
\hline
\end{tabular}

Note that $a^{*}$ seems to have similar properties in both linear and rational premium examples.

\section{Proofs}

\subsection{Proof of the Verification Theorem 4.1}

The proof is based on a representation of $v$ as the pointwise minimum of a class of "controlled" supersolutions of the HJB equation. We start with the observation that the value function satisfies a dynamic programming equation.

Lemma 6.1 After extending $v$ to the negative half-axis by $v(x)=w(x)$ for $x<0$, we have, for any stopping time $\tau$,

$$
v(x)=\sup _{\pi \in \Pi} \mathbb{E}_{x}\left[e^{-q \tau \wedge T} v\left(X_{\tau \wedge T}^{\pi}\right)+\int_{0}^{\tau \wedge T} e^{-q t} d L_{t}^{\pi}\right] .
$$

This follows by a straightforward adaptation of classical arguments (see, e.g., [7, pp. 276-277]). We will prove that $v$ is a supersolution of the HJB equation.

Lemma 6.2 The process

$$
V_{t}^{\pi}:=e^{-q(t \wedge T)} v\left(X_{t \wedge T}^{\pi}\right)+\int_{0}^{t \wedge T} e^{-q s} d L_{s}^{\pi}
$$

is a uniformly integrable (UI) supermartingale.

Proof Fix arbitrary $\pi \in \Pi, x \geq 0$ and $s, t \geq 0$ with $s<t$. The process $V_{t}^{\pi}$ is $\mathcal{F}_{t}$-measurable and is UI. Indeed, by Lemma 6.1, we have

$$
\mathbb{E}_{x}\left[V_{t}^{\pi}\right] \leq \sup _{\pi \in \Pi} \mathbb{E}_{x}\left[\mathrm{e}^{-q(t \wedge T)} v\left(X_{t \wedge T}^{\pi}\right)+\int_{0}^{t \wedge T} \mathrm{e}^{-q s} \mathrm{~d} L_{s}^{\pi}\right]=v(x) .
$$

Now by integration by parts, the non-positivity of $w$ and the non- exogeneous ruin assumption

$$
v(x) \leq \mathbb{E}_{x}\left[\int_{0}^{\infty} q \mathrm{e}^{-q s} r_{s}^{x} \mathrm{~d} s\right] \leq x+\int_{0}^{\infty} \mathrm{e}^{-q t} p\left(r_{t}^{x}\right) \mathrm{d} t \leq(A+1) x+B,
$$

where the function $r_{t}^{x}$ given in (3) satisfies (2). 
Let $W_{t}^{\pi}$ be the following value process:

$$
\begin{aligned}
& W_{s}^{\pi}:={\operatorname{ess} \sup J_{s}}_{J_{s}^{\tilde{\pi}},} J_{s}^{\tilde{\pi}}:=\mathbb{E}\left[\int_{0}^{T^{\tilde{\pi}}} \mathrm{e}^{-q u} \mathrm{~d} L_{u}^{\tilde{\pi}}+\mathrm{e}^{-q T^{\tilde{\pi}}} w\left(X_{T^{\tilde{\pi}}}^{\tilde{\pi}}\right) \mid \mathcal{F}_{S}\right], \\
& \Pi_{s}:=\left\{\tilde{\pi}=(\pi, \bar{\pi})=\left\{L_{u}^{\pi, \bar{\pi}}, u \geq 0\right\}: \bar{\pi} \in \Pi\right\}, \quad L_{u}^{\pi, \bar{\pi}}:= \begin{cases}L_{u}^{\pi}, & u \in[0, s[, \\
L_{s}^{\pi}+L_{u-s}^{\bar{\pi}}\left(X_{s}^{\pi}\right), & u \geq s,\end{cases}
\end{aligned}
$$

where $L^{\bar{\pi}}(x)$ denotes the process of cumulative dividends of the strategy $\bar{\pi}$ corresponding to the initial capital $x$.

The fact that $V^{\pi}$ is a supermartingale is a direct consequence of the following $\mathbb{P}$-a.s. relations:

(a) $V_{s}^{\pi}=W_{s}^{\pi}, \quad$ (b) $W_{s}^{\pi} \geq \mathbb{E}\left[W_{t}^{\pi} \mid \mathcal{F}_{s}\right]$, where $W^{\pi}$ is the process defined in (19).

Point (b) follows by classical arguments, since the family $\left\{J_{t}^{\tilde{\pi}}, \tilde{\pi} \in \Pi_{t}\right\}$ of random variables is upwards directed; see Neveu [19] and Avram et al. [9, Lem. 3.1(ii)] for details.

To prove (a), note that on account of the Markov property of $X^{\pi}$, it also follows that conditional on $X_{s}^{\pi},\left\{X_{u}^{\tilde{\pi}}-X_{s}^{\tilde{\pi}}, u \geq s\right\}$ is independent of $\mathcal{F}_{s}$. As a consequence, the following identity holds on the set $\left\{s<T^{\tilde{\pi}}\right\}$ :

$$
\begin{aligned}
& \mathbb{E}\left[\int_{0}^{T^{\tilde{\pi}}} \mathrm{e}^{-q u} \mathrm{~d} L_{u}^{\tilde{\pi}}+\mathrm{e}^{-q T^{\tilde{\pi}}} w\left(X_{T^{\tilde{\pi}}}^{\tilde{\pi}} \mid \mathcal{F}_{s}\right]\right. \\
& =\mathrm{e}^{-q s} \mathbb{E}_{X_{s}^{\pi}}\left[\int_{0}^{T^{\bar{\pi}}} \mathrm{e}^{-q u} \mathrm{~d} L_{u}^{\bar{\pi}}+\mathrm{e}^{-q T^{\bar{\pi}}} w\left(X_{T^{\bar{\pi}}}^{\bar{\pi}}\right)\right]+\int_{0}^{s} \mathrm{e}^{-q u} \mathrm{~d} L_{s}^{\pi} \\
& =\mathrm{e}^{-q s} v_{\bar{\pi}}\left(X_{s}^{\pi}\right)+\int_{0}^{s} \mathrm{e}^{-q u} \mathrm{~d} L_{u}^{\pi},
\end{aligned}
$$

and then we have the following representation:

$$
J_{s}^{\tilde{\pi}}=\mathrm{e}^{-q(s \wedge T)} v_{\bar{\pi}}\left(X_{s \wedge T}^{\pi}\right)+\int_{0}^{s \wedge T} \mathrm{e}^{-q u} \mathrm{~d} L_{u}^{\pi},
$$

which completes the proof on taking the essential supremum over the relevant family of strategies.

We prove that the value function $v$ is a solution of the HJB equation. We will denote by $\mathcal{G}$ the family of functions $g$ for which

$$
M^{g, T_{I}}:=\left\{\mathrm{e}^{-q\left(t \wedge T_{I}\right)} g\left(R_{t \wedge T_{I}}\right), t \geq 0\right\}, \quad T_{I}:=\inf \left\{t \geq 0: R_{t} \notin I\right\},
$$

is a supermartinagle for any closed interval $I \subset[0, \infty[$, and such that

$$
\frac{g(x)-g(y)}{x-y} \geq 1 \quad \text { for all } x>y \geq 0, \quad g(x) \geq w(x) \text { for } x<0
$$

and $g$ is ultimately dominated by some linear function. 
Lemma 6.3 We have $v \in \mathcal{G}$.

Proof Taking a strategy of not paying any dividends, by Lemma 6.2, we find that the process (20) with $g=v$ is a supermartingale. We will prove that

$$
v(x)-v(y) \geq x-y \quad \text { for all } x>y \geq 0 .
$$

Let $x>y$. Denote by $\pi^{\epsilon}(y)$ an $\epsilon$-optimal strategy for the case $X_{0}^{\pi}=y$. Then we take the strategy of paying $x-y$ immediately and subsequently following the strategy $\pi^{\epsilon}(y)$ (note that such a strategy is admissible), so that the following holds:

$$
v(x) \geq x-y+v_{\pi^{\epsilon}}(y) \geq v(y)-\epsilon+x-y .
$$

Since this inequality holds for any $\epsilon>0$, the stated lower bound follows. Linear domination of $v$ by some affine function follows from (18).

We now give the dual representations of the value function on a closed interval $I$. Assume that $\mathcal{H}_{I}$ is a family of functions $k$ for which

$$
\widetilde{M}_{t}^{k, \pi}:=\mathrm{e}^{-q\left(t \wedge \tau_{I}^{\pi}\right)} k\left(X_{t \wedge \tau_{I}^{\pi}}^{\pi}\right)+\int_{0}^{t \wedge \tau_{I}^{\pi}} \mathrm{e}^{-q s} \mathrm{~d} L_{s}^{\pi}
$$

is a UI supermartingale for $\tau_{I}^{\pi}:=\inf \left\{t \geq 0: X_{t}^{\pi} \notin I\right\}$ and

$$
k(x) \geq v(x) \quad \text { for } x \notin I .
$$

Then

$$
v(x)=\min _{k \in \mathcal{H}_{I}} k(x) \quad \text { for } x \in I .
$$

Indeed, let $\pi \in \Pi, k \in \mathcal{H}_{I}$ and $x \in I$. Then the Optional Stopping Theorem applied to the UI Dynkin martingale yields

$$
\begin{aligned}
k(x) & \geq \lim _{t \rightarrow \infty} \mathbb{E}_{x}\left[\mathrm{e}^{-q\left(\tau_{I}^{\pi} \wedge t\right)} k\left(X_{\tau_{I}^{\pi} \wedge t}^{\pi}\right)+\int_{0}^{\tau_{I}^{\pi} \wedge t} \mathrm{e}^{-q s} \mathrm{~d} L^{\pi}(s)\right] \\
& \geq \mathbb{E}_{x}\left[\mathrm{e}^{-q \tau_{I}^{\pi}} v\left(X_{\tau_{I}^{\pi}}^{\pi}\right)+\int_{0}^{\tau_{I}^{\pi}} \mathrm{e}^{-q s} \mathrm{~d} L^{\pi}(s)\right],
\end{aligned}
$$

where the convention $\exp \{-\infty\}=0$ is used.

Taking the supremum over all $\pi \in \Pi$ shows that $k(x) \geq v(x)$. Since $k \in \mathcal{H}_{I}$ was arbitrary, it follows that

$$
\inf _{k \in \mathcal{H}_{I}} k(x) \geq v(x)
$$

This inequality is in fact an equality since $v$ is a member of $\mathcal{H}_{I}$ by Lemma 6.2. The value function $v$ admits a more important representation from which the Verification Theorem 4.1 follows. 
Proposition 6.1 We have

$$
v(x)=\min _{g \in \mathcal{G}} g(x) .
$$

Proof Since $v \in \mathcal{G}$ in view of Lemma 6.3, by (23) it suffices to prove that $\mathcal{G} \subset \mathcal{H}_{[0, \infty[}$. The proof of this fact is similar to the proof of the shifting lemma [9, Lem.5.5]. For completeness, we give the main steps. Fix arbitrary $g \in \mathcal{G}, \pi \in \Pi$ and $s, t \geq 0$ with $s<t$. Note that $\widetilde{M}^{g, \pi}$ is adapted and UI by the linear growth condition and arguments in the proof of Lemma 6.2 and by [9, Sec.8]. Furthermore, the following (in)equalities hold true:

$$
\mathbb{E}\left[\widetilde{M}_{t}^{g, \pi} \mid \mathcal{F}_{s \wedge T}\right] \stackrel{(a)}{=} \lim _{n \rightarrow \infty} \mathbb{E}\left[\widetilde{M}_{t}^{g, \pi_{n}} \mid \mathcal{F}_{s \wedge T}\right] \stackrel{(b)}{\leq} \lim _{n \rightarrow \infty} \widetilde{M}_{s \wedge T}^{g, \pi_{n}} \stackrel{(c)}{=} \widetilde{M}_{s \wedge T}^{g, \pi} \stackrel{(d)}{=} \widetilde{M}_{s}^{g, \pi}
$$

where the sequence $\left(\pi_{n}\right)_{n \in \mathbb{N}}$ of strategies is defined by $\pi_{n}=\left\{L_{t}^{\pi_{n}}, t \geq 0\right\}$ with $L_{0}^{\pi_{n}}=L_{0}^{\pi}$ and

$$
\begin{aligned}
L_{u}^{\pi_{n}} & := \begin{cases}\sup \left\{L_{v}^{\pi}: v<u, v \in \mathbb{T}_{n}\right\}, & 0<u<T, \\
L_{T-}^{\pi_{n}}, & u \geq T,\end{cases} \\
\mathbb{T}_{n} & :=\left(\left\{t_{k}:=s+(t-s) \frac{k}{2^{n}}, k \in \mathbb{Z}\right\} \cup\{0\}\right) \cap \mathbb{R}_{+},
\end{aligned}
$$

where the above $T$ is calculated for the strategy $\pi$. Since $s$ and $t$ are arbitrary, it follows that $\widetilde{M}^{g, \pi}$ is a supermartingale, which will complete the proof.

Points (a), (c) and (d) follow from the Monotone and Dominated Convergence Theorems. To prove (b), let $T_{i}:=T \wedge t_{i}$, denote $\widetilde{M}^{g, \pi_{n}}=M, L^{\pi_{n}}=L$ and observe that

$$
\begin{aligned}
& M_{t}-M_{s}=\sum_{i=1}^{2^{n}} Y_{i}+\sum_{i=1}^{2^{n}} Z_{i}, \text { with } \\
& Y_{i}:=\mathrm{e}^{-q T_{i}} g\left(X_{T_{i}-}\right)-\mathrm{e}^{-q T_{i-1}} g\left(X_{T_{i-1}}\right), \\
& Z_{i}:=\mathrm{e}^{-q T_{i}}\left(g\left(X_{T_{i}}\right)-g\left(X_{T_{i}-}\right)+\Delta L_{T_{i}}\right) \mathbb{I}_{\left\{\Delta L_{T_{i}}>0\right\}} .
\end{aligned}
$$

The strong Markov property of $R$ and the definition of $X^{\pi}$ imply

$$
\begin{aligned}
\mathbb{E}\left[Y_{i} \mid \mathcal{F}_{T_{i-1}}\right] & =\mathrm{e}^{-q T_{i-1}} \mathbb{E}\left[\mathrm{e}^{-q\left(T_{i}-T_{i-1}\right)} g\left(X_{T_{i-}}\right)-g\left(X_{T_{i-1}}\right) \mid \mathcal{F}_{T_{i-1}}\right] \\
& =\mathrm{e}^{-q T_{i-1}} \mathbb{E}_{X_{T_{i-1}}}\left[\mathrm{e}^{-q \tau_{i}} g\left(R_{\tau_{i}}\right)-g\left(R_{0}\right)\right],
\end{aligned}
$$

with $\tau_{i}:=T_{i} \circ \theta_{T_{i-1}}$, where $\theta$ denotes the shift operator. The right-hand side of (25) is non-positive because $g \in \mathcal{G}$. Furthermore, it follows from (21) that all the $Z_{i}$ are non-positive. The tower property of conditional expectation then yields

$$
\mathbb{E}\left[M_{t}-M_{s} \mid \mathcal{F}_{s}\right] \leq 0
$$

This establishes inequality (b), and the proof is complete. 
Proof of the Verification Theorem 4.1 Since $v_{\pi}$ is absolutely continuous and dominated by an affine function, $v_{\pi}$ is in the domain of the full generator of $R$. This means that the process

$$
v_{\pi}\left(R_{t \wedge T_{I}}\right) \mathrm{e}^{-\int_{0}^{t \wedge T_{I}} \frac{\mathcal{A} v_{\pi}\left(R_{S}\right)}{v_{\pi}\left(R_{S}\right)} \mathrm{d} s}
$$

is a martingale for any closed interval $I \in\left[0, \infty\left[\right.\right.$. By (9), it follows that $v_{\pi} \in \mathcal{G}$, which completes the proof.

\subsection{Proof of Lemma 4.1}

Take any $x \geq 0$. Then fix $a>0$ such that $x<a$. From the definition of $W_{q}$ given in (7), conditioning on the first claim arrival time $\sigma_{1}$, we obtain

$$
W_{q}(x)=\mathrm{e}^{-(\lambda+q) h} W_{q}\left(r_{h}^{x}\right)+\lambda \int_{0}^{h} \int_{0}^{r_{t}^{x}} W_{q}\left(r_{t}^{x}-z\right) \mathrm{d} F(z) \mathrm{e}^{-(\lambda+q) t} \mathrm{~d} t,
$$

for $h$ small enough, so that $r_{h}^{x}<a$. As $h \downarrow 0$, we find that $W_{q}$ is right-continuous at $x$. Moreover, rearranging terms in (26) leads to

$$
\begin{aligned}
\frac{W_{q}\left(r_{h}^{x}\right)-W_{q}(x)}{r_{h}^{x}-x}= & \frac{1-\mathrm{e}^{-(\lambda+q) h}}{h} \frac{h}{r_{h}^{x}-x} W_{q}\left(r_{h}^{x}\right) \\
& -\frac{h}{r_{h}^{x}-x} \frac{\lambda}{h} \int_{0}^{h} \int_{0}^{r_{t}^{x}} W_{q}\left(r_{t}^{x}-z\right) \mathrm{d} F(z) \mathrm{e}^{-(\lambda+q) t} \mathrm{~d} t .
\end{aligned}
$$

Letting $h \downarrow 0$ we conclude that $W_{q}$ is right-differentiable with derivative

$$
W_{q,+}^{\prime}(x)=\frac{1}{p(x)}\left((\lambda+q) W_{q}(x)-\lambda \int_{0}^{x} W_{q}(x-z) \mathrm{d} F(z)\right) .
$$

Now take any $x>0$. Equation (26) can be rewritten as

$$
W_{q}\left(\tilde{r}_{0}^{x}\right)=\mathrm{e}^{-(\lambda+q) h} W_{q}(x)+\lambda \int_{0}^{h} \int_{0}^{\tilde{r}_{t}^{x}} W_{q}\left(\tilde{r}_{t}^{x}-z\right) \mathrm{d} F(z) \mathrm{e}^{-(\lambda+q) t} \mathrm{~d} t,
$$

where $\tilde{r}^{x}$ is a solution to the backward equation $\mathrm{d} \tilde{r}_{t}^{x}=p\left(\tilde{r}_{s}^{x}\right) \mathrm{d} s, \tilde{r}_{h}^{x}=x$. We take $h$ small enough, so that $\tilde{r}_{0}^{x} \geq 0$. We thus get left continuity and

$$
\begin{aligned}
\frac{W_{q}(x)-W_{q}\left(\tilde{r}_{0}^{x}\right)}{x-\tilde{r}_{0}^{x}}= & \frac{1-\mathrm{e}^{-(\lambda+q) h}}{h} \frac{h}{x-\tilde{r}_{0}^{x}} W_{q}(x) \\
& -\frac{h}{x-\tilde{r}_{0}^{x}} \frac{\lambda}{h} \int_{0}^{h} \int_{0}^{\tilde{r}_{t}^{x}} W_{q}\left(\tilde{r}_{t}^{x}-z\right) \mathrm{d} F(z) \mathrm{e}^{-(\lambda+q) t} \mathrm{~d} t .
\end{aligned}
$$


Letting $h \downarrow 0$ we see that $W_{q}$ is left-differentiable with derivative

$$
W_{q,-}^{\prime}(x)=\frac{1}{p(x)}\left((\lambda+q) W_{q}(x)-\lambda \int_{0}^{x-} W_{q}(x-z) \mathrm{d} F(z)\right) .
$$

Since $F$ is absolutely continuous, (27) and (28) imply that $W_{q}$ is continuously differentiable and satisfies (14). Using the same arguments and definition (8), one can show that the function $G_{q, w}$ is continuously differentiable and satisfies (15). This completes the proof.

\subsection{On the Value Function for the Barrier Strategy}

Note that for the barrier strategy until the first hitting of the barrier $a$, the regulated process $X^{\pi_{a}}$ behaves like the process $R$. By the strong Markov property of the PDMP $R_{t}$ and by (7) for $x \in[0, a]$, we have

$$
v_{a}(x)=\frac{W_{q}(x)}{W_{q}(a)} v_{a}(a)+\mathbb{E}_{x}\left[\mathrm{e}^{-q \tau_{0}^{-}} w\left(R_{\tau_{0}^{-}}\right) \mathbb{I}_{\left\{\tau_{0}^{-}<\tau_{a}^{+}\right\}}\right] .
$$

Moreover, again using the strong Markov property, we can derive

$$
\mathbb{E}_{x}\left[\mathrm{e}^{-q \tau_{0}^{-}} w\left(R_{\tau_{0}^{-}}\right) \mathbb{I}_{\left\{\tau_{0}^{-}<\tau_{a}^{+}\right\}}\right]=G_{q, w}(x)-G_{q, w}(a) \frac{W_{q}(x)}{W_{q}(a)} .
$$

Hence

$$
v_{a}(x)=\frac{W_{q}(x)}{W_{q}(a)}\left(v_{a}(a)-G_{q, w}(a)\right)+G_{q, w}(x) .
$$

We will prove that

$$
v_{a}^{\prime}(a)=1,
$$

from which the assertion of Theorem 4.2 immediately follows.

Note that for the barrier strategy $a$, we have

$$
v_{a}(x)=x-a+v_{a}(a) \quad \text { for } x>a .
$$

Take any $a>0$ and $x \in\left[0, a\left[\right.\right.$. From the definition of $v_{a}$ given in (6) and fixed $a$, conditioning on the first claim arrival time $\sigma_{1}$, we obtain

$$
\begin{aligned}
v_{a}(x)= & \mathrm{e}^{-(\lambda+q) h} v_{a}\left(r_{h}^{x}\right)+\lambda \int_{0}^{h} \int_{0}^{r_{t}^{x}} v_{a}\left(r_{t}^{x}-z\right) d F(z) \mathrm{e}^{-(\lambda+q) t} \mathrm{~d} t \\
& +\lambda \int_{0}^{h} \int_{r_{t}^{x}}^{\infty} w\left(r_{t}^{x}-z\right) \mathrm{d} F(z) \mathrm{e}^{-(\lambda+q) t} \mathrm{~d} t,
\end{aligned}
$$


where $h$ is small enough (so that $\left.r_{h}^{x} \in\right] 0, a$ [). Letting $h \downarrow 0$ we find that $v_{a}$ is rightcontinuous at $x$ for all $x \in[0, a$ [. Moreover, rearranging terms in (32) leads to

$$
\begin{aligned}
\frac{v_{a}\left(r_{h}^{x}\right)-v_{a}(x)}{r_{h}^{x}-x}= & \frac{1-\mathrm{e}^{-(\lambda+q) h}}{h} \frac{h}{r_{h}^{x}-x} v_{a}\left(r_{h}^{x}\right) \\
& -\frac{h}{r_{h}^{x}-x} \frac{\lambda}{h} \int_{0}^{h} \int_{0}^{r_{t}^{x}} v_{a}\left(r_{t}^{x}-z\right) \mathrm{d} F(z) \mathrm{e}^{-(\lambda+q) t} \mathrm{~d} t \\
& +\frac{h}{r_{h}^{x}-x} \frac{\lambda}{h} \int_{0}^{h} \int_{r_{t}^{x}}^{\infty} w\left(r_{t}^{x}-z\right) \mathrm{d} F(z) \mathrm{e}^{-(\lambda+q) t} \mathrm{~d} t .
\end{aligned}
$$

Letting $h \downarrow 0$ we conclude that $v_{a}$ is right-differentiable on $[0, a$ [ with derivative satisfying

$$
p(x) v_{a,+}^{\prime}(x)=(\lambda+q) v_{a}(x)-\lambda \int_{0}^{x} v_{a}(x-z) \mathrm{d} F(z)-\lambda \int_{x}^{\infty} w(x-z) \mathrm{d} F(z) .
$$

Now take any $x \in] 0, a]$. Equation (32) can be rewritten as

$$
\begin{aligned}
v_{a}\left(\tilde{r}_{0}^{x}\right)= & \mathrm{e}^{-(\lambda+q) h} v_{a}(x)+\lambda \int_{0}^{h} \int_{0}^{\tilde{r}_{t}^{x}} v_{a}\left(\tilde{r}_{t}^{x}-z\right) \mathrm{d} F(z) \mathrm{e}^{-(\lambda+q) t} \mathrm{~d} t \\
& +\lambda \int_{0}^{h} \int_{\tilde{r}_{t}^{x}}^{\infty} w\left(\tilde{r}_{t}^{x}-z\right) \mathrm{d} F(z) \mathrm{e}^{-(\lambda+q) t} \mathrm{~d} t,
\end{aligned}
$$

where $\tilde{r}^{x}$ is a solution to the backward equation $\mathrm{d} \tilde{r}_{t}^{x}=p\left(\tilde{r}_{s}^{x}\right) \mathrm{d} s, \tilde{r}_{h}^{x}=x$. We take $h$ small enough, so that $\tilde{r}_{0}^{x} \geq 0$. We thus get left continuity on $\left.] 0, a\right]$ and

$$
\begin{aligned}
\frac{v_{a}(x)-v_{a}\left(\tilde{r}_{h}^{x}\right)}{x-\tilde{r}_{h}^{x}}= & \frac{1-\mathrm{e}^{-(\lambda+q) h}}{h} \frac{h}{x-\tilde{r}_{h}^{x}} v_{a}\left(\tilde{r}_{h}^{x}\right) \\
& -\frac{h}{x-\tilde{r}_{h}^{x}} \frac{\lambda}{h} \int_{0}^{h} \int_{0}^{\tilde{r}_{t}^{x}} v_{a}\left(\tilde{r}_{t}^{x}-z\right) \mathrm{d} F(z) \mathrm{e}^{-(\lambda+q) t} \mathrm{~d} t \\
& +\frac{h}{x-\tilde{r}_{h}^{x}} \frac{\lambda}{h} \int_{0}^{h} \int_{\tilde{r}_{t}^{x}}^{\infty} w\left(\tilde{r}_{t}^{x}-z\right) \mathrm{d} F(z) \mathrm{e}^{-(\lambda+q) t} \mathrm{~d} t .
\end{aligned}
$$

Letting $h \downarrow 0$ we infer that $v_{a}$ is left-differentiable on $\left.] 0, a\right]$ with derivative

$$
p(x) v_{a,-}^{\prime}(x)=(\lambda+q) v_{a}(x)-\lambda \int_{0}^{x-} v_{a}(x-z) \mathrm{d} F(z)-\lambda \int_{x-}^{\infty} w(x-z) \mathrm{d} F(z) .
$$

Under the assumption that $F$ is absolutely continuous, the function $v_{a}$ is differentiable on $] 0, a$. Now we will prove that it is differentiable at $x=a$. Take $x=a$. Then from the definition of $v_{a}$, for $x=a$, conditioning on the first claim arrival time, we obtain 


$$
\begin{aligned}
v_{a}(a)= & \mathrm{e}^{-(q+\lambda) h} v_{a}(a)+\mathrm{e}^{-\lambda h} \int_{0}^{h} \mathrm{e}^{-q t} p(a) \mathrm{d} t+\lambda \int_{0}^{h} \int_{0}^{a} v_{a}(a-z) \mathrm{d} F(z) \mathrm{e}^{-(q+\lambda) t} \mathrm{~d} t \\
& +\lambda \int_{0}^{h} \int_{a}^{\infty} w(a-z) \mathrm{d} F(z) \mathrm{e}^{-(q+\lambda) t} \mathrm{~d} t+\lambda p(a) \int_{0}^{h} \int_{0}^{t} \mathrm{e}^{-q s} \mathrm{e}^{-\lambda t} \mathrm{~d} s \mathrm{~d} t .
\end{aligned}
$$

Differentiating (35) with respect to $h$ and setting $h=0$ gives

$$
0=-(\lambda+q) v_{a}(a)+\lambda \int_{0}^{a} v(a-z) \mathrm{d} F(z)+\lambda \int_{a}^{\infty} w(a-z) \mathrm{d} F(z)+p(a) .
$$

By setting $x=a$ in (34) and using (36), we get $v_{a,-}^{\prime}(a)=1$. This together with (31) proves that $v_{a}$ has a derivative at $a$ and (30) holds.

\subsection{Proofs of Necessary and Sufficient Conditions for Optimality of the Barrier Strategy}

Proof of Theorem 4.3 To prove sufficiency, we need to show that $v_{a^{*}}$ satisfies the conditions of the Verification Theorem 4.1. From Theorem 4.2, it follows that $v_{a^{*}}$ is ultimately linear. Moreover, by the choice of the optimal barrier $a^{*}$, we know that $v_{a^{*}}^{\prime}(x) \geq 1$. Finally, by definition of $W_{q}$ and $G_{q, w}$ and the strong Markov property of the risk process $R$, it follows that

$$
\mathrm{e}^{-q(t \wedge T)} W_{q}\left(R_{t \wedge T \wedge \tau_{a^{*}}^{+}}\right), \quad \mathrm{e}^{-q(t \wedge T)} G_{q, w}\left(R_{t \wedge T}\right)
$$

are martingales. Hence

$$
\mathrm{e}^{-q(t \wedge T)} v_{a^{*}}\left(R_{t \wedge T \wedge \tau_{a^{*}}^{+}}\right)
$$

is a martingale. This means that $v_{a^{*}}$ is in the domain of the full generator of $R$ stopped on exiting $\left[0, a^{*}\right]$ and that $(\mathcal{A}-q) v_{a^{*}}(x)=0$ for $x \leq a^{*}$.

To prove necessity, we assume that condition (12) is not satisfied. By the continuity of the function $x \mapsto(\mathcal{A}-q) v_{a^{*}}(x)$, there exists an open and bounded interval $\mathrm{J} \subset$ ] $a^{*}, \infty\left[\right.$ such that $(\mathcal{A}-q) v_{a^{*}}(x)>0$ for all $x \in \mathrm{J}$. Let $\tilde{\pi}$ be the strategy of paying nothing if the reserve process $X^{\tilde{\pi}}$ takes a value in $\mathrm{J}$, and following the strategy $\pi_{a^{*}}$ otherwise. If we extend $v_{a^{*}}$ to the negative half-axis by $v_{a^{*}}(x)=w(x)$ for $x<0$, we have

$$
v_{\tilde{\pi}}(x)= \begin{cases}\mathbb{E}_{x}\left[e^{-q T_{\mathrm{J}}} v_{a^{*}}\left(R_{T_{\mathrm{J}}}\right)\right], & x \in \mathrm{J}, \\ v_{a^{*}}(x), & x \notin \mathrm{J},\end{cases}
$$

where $T_{\mathrm{J}}$ is defined by (20).

By the Optional Stopping Theorem applied to the process $\mathrm{e}^{-q t} v_{a^{*}}\left(R_{t}\right)$, for all $x \in \mathrm{J}$, we obtain

$$
v_{\tilde{\pi}}(x)=\mathbb{E}_{x}\left[\mathrm{e}^{-q T_{\mathrm{J}}} v_{a^{*}}\left(R_{T_{\mathrm{J}}}\right)\right]=v_{a^{*}}(x)+\mathbb{E}_{x}\left[\int_{0}^{T_{\mathrm{J}}}(\mathcal{A}-q) v_{a^{*}}\left(R_{S}\right) \mathrm{d} s\right]>v_{a^{*}}(x) .
$$


This leads to a contradiction with the optimality of the strategy $\pi_{a^{*}}$, and the proof is complete.

Proof of Theorem 4.4 In the first step, we will show that

$$
\lim _{y \uparrow x}(\mathcal{A}-q)\left(v_{a^{*}}-v_{x}\right)(y) \leq 0 \quad \text { for all } x>a^{*}
$$

Let $x>a^{*}$. By the Dominated Convergence Theorem, we obtain

$$
\begin{aligned}
\lim _{y \uparrow x}(\mathcal{A}-q)\left(v_{a^{*}}-v_{x}\right)(y)= & p(x)\left(v_{a^{*}}^{\prime}-v_{x}^{\prime}\right)(x)-q\left(v_{a^{*}}-v_{x}\right)(x) \\
& +\int_{0}^{\infty}\left[\left(v_{a^{*}}-v_{x}\right)(x-z)-\left(v_{a^{*}}-v_{x}\right)(x)\right] \lambda F(\mathrm{~d} z) .
\end{aligned}
$$

By (10), we have:

i. $\left(v_{a^{*}}^{\prime}-v_{x}^{\prime}\right)(x)=0$.

ii. $\left(v_{a^{*}}^{\prime}-v_{x}^{\prime}\right)(b)=W_{q}^{\prime}(b)\left(H_{q}^{\prime}\left(a^{*}\right)-H_{q}^{\prime}(x)\right) \geq 0$ for $b \in\left[0, a^{*}\right]$ by the definition of $a^{*}$.

iii. $\left(v_{a^{*}}^{\prime}-v_{x}^{\prime}\right)(u)=W_{q}^{\prime}(u)\left(H_{q}^{\prime}(u)-H_{q}^{\prime}(x)\right) \geq 0$ for $u \in\left[a^{*}, x\right]$ by the assumption (13).

iv. $\left(v_{a^{*}}-v_{x}\right)\left(a^{*}\right) \geq 0$, thus by iii, $\left(v_{a^{*}}-v_{x}\right)(x) \geq 0$.

v. $\left(v_{a^{*}}-v_{x}\right)(x-z) \leq\left(v_{a^{*}}-v_{x}\right)(x)$ for all $z \geq 0$ by ii and iii.

Thus, we have shown (37).

Now assume that (12) does not hold. Then there exists $x>a^{*}$ such that $(\mathcal{A}-$ $q) v_{a^{*}}(x)>0$. By the continuity of $(\mathcal{A}-q) v_{a^{*}}$, we deduce that $\lim _{y \uparrow x}(\mathcal{A}-q) v_{x}(y)>$ 0 , which contradicts (37).

Proof of Theorem 4.5 In view of Theorem 4.3, it follows that to prove optimality of $v_{a^{*}}$, we need to verify that $g(x) \leq 0$ for $x>a^{*}$, where

$$
g(x):=\mathcal{A} v_{a^{*}}(x)-q v_{a^{*}}(x) .
$$

Recall that

$g\left(x+a^{*}\right)=p\left(x+a^{*}\right)-q v_{a^{*}}\left(a^{*}\right)-q x+\lambda \int_{0}^{\infty}\left(v_{a^{*}}\left(x+a^{*}-y\right)-v_{a^{*}}\left(x+a^{*}\right)\right) f(y) \mathrm{d} y$.

The desired assertion follows once the following three facts are verified: (i) $g$ is concave on $\mathbb{R}_{+} \backslash\{0\}$, (ii) $g\left(a^{*}\right)=0$ and (iii) $g^{\prime}\left(a^{*}\right)=0$.

To show (i) recall that $g(x)=0$ for all $x \leq a^{*}$ (see the proof of Prop. 4.3). Moreover, denoting $k(x, y):=v_{a^{*}}\left(x+a^{*}-y\right)-v_{a^{*}}\left(x+a^{*}\right)$ and noting that $\frac{\partial^{2}}{\partial x^{2}} k(x, y)=$ $\frac{\partial^{2}}{\partial y^{2}} k(x, y)$, we have 


$$
\begin{aligned}
\frac{\partial^{2}}{\partial x^{2}} \int_{0}^{\infty} k(x, y) f(y) \mathrm{d} y & =\int_{0}^{\infty} \frac{\partial^{2}}{\partial y^{2}} k(x, y) f(y) \mathrm{d} y \\
& =\left.\frac{\partial}{\partial y} k(x, y) f(y)\right|_{0} ^{\infty}-\left.k(x, y)\right|_{0} ^{\infty}+\int_{0}^{\infty} k(x, y) f^{\prime \prime}(y) \mathrm{d} y \leq 0
\end{aligned}
$$

since $v_{a^{*}}\left(x+a^{*}-y\right)-v_{x+a^{*}}\left(a^{*}\right) \leq 0, f^{\prime \prime}(y) \geq 0, \frac{\partial}{\partial y} k(x, 0)=v_{a^{*}}^{\prime}\left(x+a^{*}\right)=1$ and $f(0)=0$.

Point (ii) is straightforward, and (iii) follows from the fact that $g^{\prime}(x)=0$ for any $x<a^{*}$ and $g$ is continuously differentiable.

Proof of Theorem 4.6 Let $g$ be defined by (38). Recall that by the definition of $a^{*}$, we have $g\left(a^{*}\right)=0$. Moreover, for $x \geq a^{*}$, we have

$$
g^{\prime}(x)=p^{\prime}(x)+\lambda \int_{0}^{x} v_{a^{*}}(y) f^{\prime}(x-y) \mathrm{d} y-(q+\lambda) .
$$

Note that in the case of $w \equiv 0, v_{x} \geq 0$ for all $x \geq 0$. Thus, by assumption $g^{\prime}(x) \leq 0$ for $x \geq 0$ and by Theorem 4.3 , the strategy $\pi_{a^{*}}$ is optimal.

\section{Conclusions}

In this paper, we solved the dividend problem with a penalty function at ruin. We found some sufficient and necessary conditions for a barrier strategy to be optimal. Unfortunately, some of them, like (12) and (13), may be difficult to verify. Moreover, we analyzed only single barrier strategies. Therefore, one can consider "multi-bands strategies" (see [9]). It would also be interesting to consider the effect of adding fixed transaction costs that have to be paid when dividends are being paid. In the next step, it would be reasonable to examine the so-called "dual model" with a negative premium function and positive jumps. In such a model, the premiums are regarded as costs and claims are viewed as profits. Such a model might be appropriate for a company that specializes in inventions and discoveries (see [20]). However, we leave these points for future research.

Acknowledgments This work is partially supported by the National Science Centre under the grant DEC-2013/09/B/ST1/01778. The second author kindly acknowledges partial support by the project RARE -318984, a Marie Curie IRSES Fellowship within the 7th European Community Framework Programme.

Open Access This article is distributed under the terms of the Creative Commons Attribution 4.0 International License (http://creativecommons.org/licenses/by/4.0/), which permits unrestricted use, distribution, and reproduction in any medium, provided you give appropriate credit to the original author(s) and the source, provide a link to the Creative Commons license, and indicate if changes were made.

\section{References}

1. De Finetti, B.: Su un'impostazione alternativa della teoria collettiva del rischio. Trans. XV Intern. Congress Act 2, 433-443 (1957)

2. Gerber, H.U., Shiu, E.S.W.: Optimal dividends: analysis with Brownian motion. N. Am. Actuar. J. 8, 1-20 (2004) 
3. Jeanblanc, M., Shiryaev, A.N.: Optimization of the flow of dividends. Russian Math. Surveys 50, 257-277 (1995)

4. Irbäck, J.: Asymptotic theory for a risk process with a high dividend barrier. Scand. Actuarial J. 2, 97-118 (2003)

5. Zhou, X.: On a classical risk model with a constant dividend barrier. N. Am. Actuar. J. 9, 1-14 (2005)

6. Asmussen, S., Højgaard, B., Taksar, M.: Optimal risk control and dividend distribution policies. Example of excess-of-loss reinsurance for an insurance corporation. Finance Stoch. 4, 299-324 (2000)

7. Azcue, P., Muler, N.: Optimal reinsurance and dividend distribution policies in the Cramér-Lundberg model. Math. Finance 15, 261-308 (2005)

8. Avram, F., Palmowski, Z., Pistorius, M.R.: On the optimal dividend problem for a spectrally negative Lévy process. Ann. Appl. Probab. 17, 156-180 (2007)

9. Avram, F., Palmowski, Z., Pistorius, M.R.: On Gerber-Shiu functions and optimal dividend distribution for a Lévy risk-process in the presence of a penalty function. Ann. Appl. Probab. 25(4), 1868-1935 (2015)

10. Segerdahl, C.: Über einige risikotheoretische Fragestellungen. Skandinavisk Aktuartidsskrift 25, 43$83(1942)$

11. Kyprianou, A., Palmowski, Z.: Distributional study of De Finetti's dividend problem for a general Lévy insurance risk process. J. Appl. Probab. 44, 428-443 (2007)

12. Loeffen, R.: On optimality of the barrier strategy in de Finetti's dividend problem for spectrally negative Lévy processes. Ann. Appl. Probab. 18, 1669-1680 (2008)

13. Loeffen, R.: An optimal dividends problem with transaction costs for spectrally negative Lévy processes. Insur. Math. Econ. 45, 41-48 (2009)

14. Loeffen, R., Renaud, J.-F.: De Finetti's optimal dividends problem with an affine penalty function at ruin. Insur. Math. Econ. 46, 98-108 (2009)

15. Schmidli, H.: Stochastic Control in Insurance. Springer, London (2008)

16. Davis, M.H.A.: Markov Models and Optimization, Monographs on Statistics and Applied Probability. Chapman \& Hall, London (1993)

17. Rolski, T., Schmidli, H., Schmidt, V., Teugels, J.: Stochastic Processes for Insurance and Finance. Wiley, Chichester (1999)

18. Albrecher, H., Constantinescu, C., Palmowski, Z., Regensburger, G., Rosenkranz, M.: Exact and asymptotic results for insurance risk models with surplus-dependent premiums. SIAM J. Appl. Math. 73, 47-66 (2013)

19. Neveu, J.: Discrete Parameter Martingales. North-Holland, Amsterdam (1975)

20. Avanzi, B., Gerber, H.U.: Optimal dividends in the dual model with diffusion. Astin Bull. 38, 653-667 (2008) 